\title{
Evidence for atmospheric pollution across the Permian- Triassic transition
}

\author{
Peter A. Hochuli ${ }^{1}$, Elke Schneebeli-Hermann ${ }^{1}$, Gunn Mangerud ${ }^{2}$, Hugo Bucher ${ }^{1}$ \\ ${ }^{1}$ Institute and Museum of Palaeontology, University of Zurich, Karl Schmid-Str. 4, CH-8006 \\ Zurich, Switzerland \\ ${ }^{2}$ Department of Earth Science, University of Bergen, Postboks 7803, N-5020 Bergen, Norway
}

\section{DATA REPOSITORY}

\section{Plate 1}

Deformed Spores

*England Finder coordinates; scale bar $20 \mu \mathrm{m}$

$1 \quad$ Uvaesporites imperialis (normal); 7128/12-U-1 - 112.76m; J53/1*

2,6 Uvaesporites imperialis (normal and deformed); 7128/12-U-1 - 112.76m; J51*

3 Uvaesporites sp. (folded surface); 7128/12-U-1 - 115.9m; J53/1*

4, 5 Uvaesporites imperialis (deformed trilete mark and shape); 7128/12-U-1 114.37m; $\mathrm{G} 34 / 4 *$

7 Uvaesporites imperialis (reduced inner body); 7129/10-U-1 - 63.48m; S40/1*

8,9 Uvaesporites imperialis (deformed trilete mark); 7128/12-U-1 - 108.79m; E50/3*

10 Densoisporites sp. (partly brocken, deformed trilete mark); 7128/12-U-1 - 112.76m; L60/2*

11 Trilete spore (?Uvaesporites sp., deformed trilete mark); 7128/12-U-1 114.37m; G43/4*

12 Spore tetrad and trilete spore with deformed trilete mark; 7128/12-U-1 - 94.88m; L30/2*

13 Trilete spore (?Uvaesporites sp., deformed trilete mark); 7128/12-U-1 - 94.88m; $\mathrm{E} 37 / 2 *$ 
Plate 1
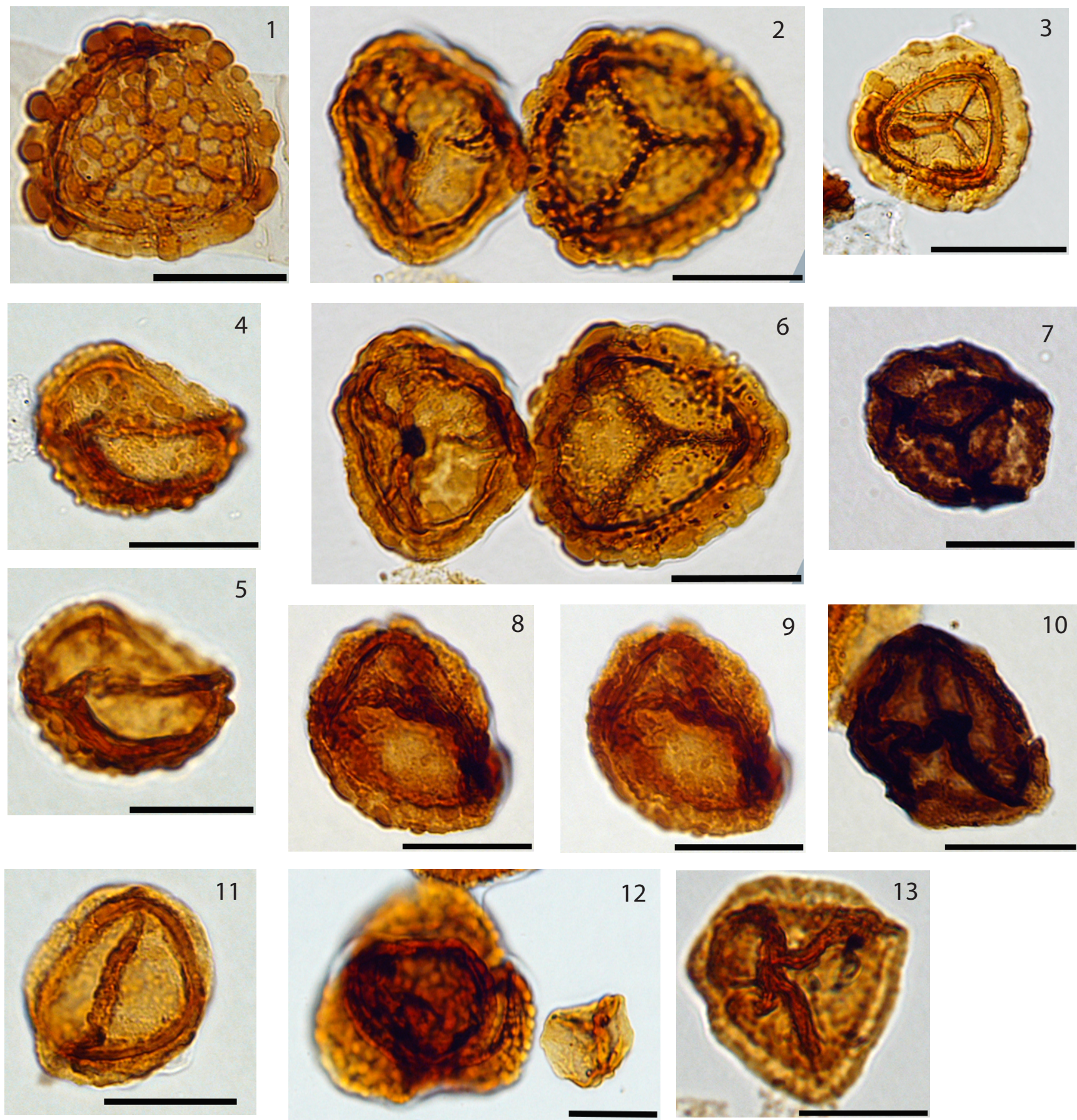


\section{Plate 2}

\section{Tetrads}

*England Finder coordinates; scale bar $20 \mu \mathrm{m}$

$1 \quad$ Uvaesporites sp. (tetrad); 7128/12-U-1 - 115.9m; H41/1*

2,3 Densoisporites sp. (tetrad); 7128/12-U-1 - 108.79m; E61*

4 Densoisporites sp. (welded tetrad); 7128/12-U-1 - 105.69m; P61/3*

5 Trilete spores (tetrad with unequal spore size); 7129/10-U-1 - 63.48m; Q52/3*

6 Ephedripites spp. (polyade); 7128/12-U-1 - 81.87m; G70/2*

7 Tetrad with one aborted spore; 7129/10-U-1 - 63.48m; Q41/3*

8 Trilete spores (double tetrad); 7128/12-U-1 - 94.88m; E54/4*

9 Ephedripites spp. (tetrad); 7128/12-U-1 - 94.88m; E36*

10 deformed tetrad (welded tetrad); 7128/12-U-1 - 105.69m; P33*

11 Bisaccate pollen grains (tetrad); 7128/12-U-1 - 84.74m; Q36/3* 
Plate 2
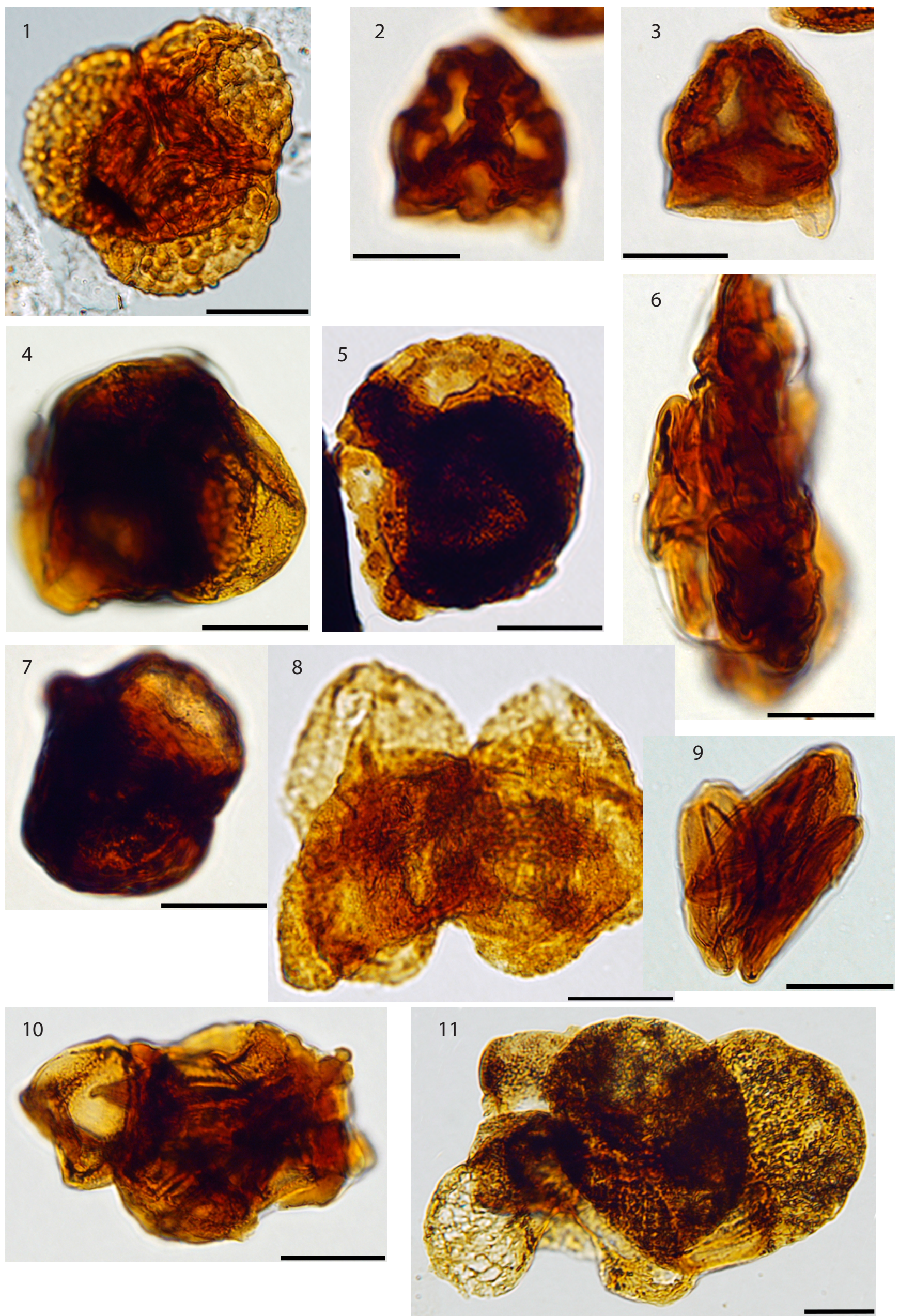


\section{Plate 3}

\section{Deformed bisaccate pollen grains}

*England Finder coordinates; scale bar $20 \mu \mathrm{m}$

$1 \quad$ Bisaccate pollen grain (?Klausipollenites sp. with reduced and deformed saccus); 7128/12-U-1 - 116.47m; O57/3*

2 Taeniate bisaccate pollen grain (deformed taeniae and sacci of unequal size) 7128/12U-1 - 119.09m; M54/4*

3 Taeniate bisaccate pollen grain (deformed - oblique taeniae); 7128/12-U-1 - 112.76m; $\mathrm{L} 36 / 2^{*}$

4 Taeniate bisaccate pollen grain (deformed central body and sacci); 7128/12-U-1 $119.09 ; 19.7 / 127.2 *$

5 Bisaccate pollen grain (folded and deformed central body); 7128/12-U-1 - 112.76m; Q57/1*

6 Bisaccate pollen grain (one abnormal saccus); 7129/10-U-1 - 65.68m; U52/2*

7 Taeniate bisaccate pollen grain (deformed taeniae and sacci of unequal size); 7128/12U-1 - 112.76m; L36/1*

8 Bisaccate pollen grain (?Klausipollenites sp., deformed and modified number of sacci 7128/12-U-1 - 87.70m; N26/1*

9 Taeniate bisaccate pollen grain (?Striatoabieites sp., (reduced taeniate and unequal size of sacci); 7128/12-U-1 - 119.09m; Q53/2*

10 Bisaccate pollen grain (deformed central body and sacci) 7129/10-U-1 - 65.68m; M47/4*

11 Taeniate bisaccate pollen grain (deformed taeniate and sacci) 7128/12-U-1 - 119.09m; L56/2*

12 Klausipollenites sp. (three grains with deformed sacci); 7128/12-U-1 - 105.69m; G31*

12 Bisaccate pollen grain (deformed sacci of unequal size); 7129/10-U-1 - 65.68m; M27/4*

14 Potonieisporites sp. (asymmetric saccus); 7128/12-U-1 - 141.09m; H28* 
Plate 3

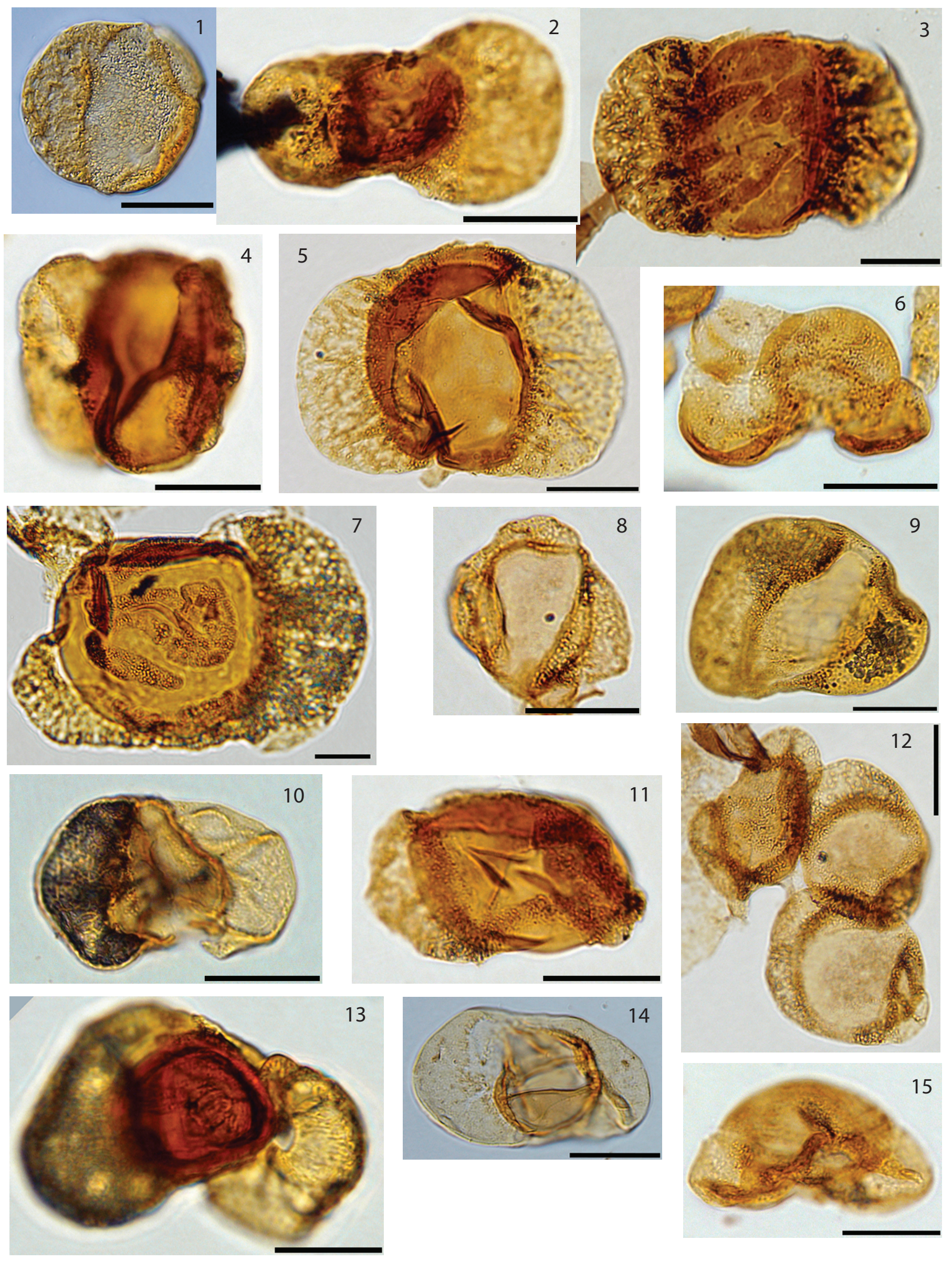




\section{Plate 4}

\section{Darkened sporomorphs}

*England Finder coordinates; scale bar $20 \mu \mathrm{m}$

1 Bisaccate pollen grains (dark + translucent); 7128/12-U-1 - 81.87m; E46/1*

2 Uvaesporites imperialis (dark + translucent); 7128/12-U-1 - 114.37m; G39/4*

34 Trilete spores - (dark + translucent); 7128/12-U-1 - 108.79m; S38/1*

42 tetrads (dark + translucent); 7129/10-U-1 - 63.48m; Q45/4*

$5 \quad$ Uvaesporites spp. (dark + translucent); 7129/10-U-1 - 63.48m; V61/1*

6 Tetrad (dark, "mazerated"); 7129/10-U-1 - 63.48m; F60/4*

$7 \quad$ Endosporites papillosus (translucent) and trilete spore (cf. Uvaesporites sp., dark) 7128/12-U-1 - 105.69m; M66/3*

8 Endosporites papillosus (dark); 7129/10-U-1 - 63.48m; J30/2*

9 Densoisporites sp. (deformed and darkened); 7128/12-U-1 - 105.69m; G34*

10 Uvaesporites sp. (dark); 7128/12-U-1 - 105.69m; E44/3*

11 Taeniate bisaccate (dark); 7128/12-U-1 - 81.87m; E29/1*

12 Tetrad (dark); 7129/10-U-1 - 63.48m; R52/2*

13 Fungal remains (dark) + acritarchs (translucent); 7129/10-U-1 - 63.48m; Q46/4*

14 Lueckisporites sp. (dark); 7129/10-U-1 - 63.48m; R51/4* 
Plate 4
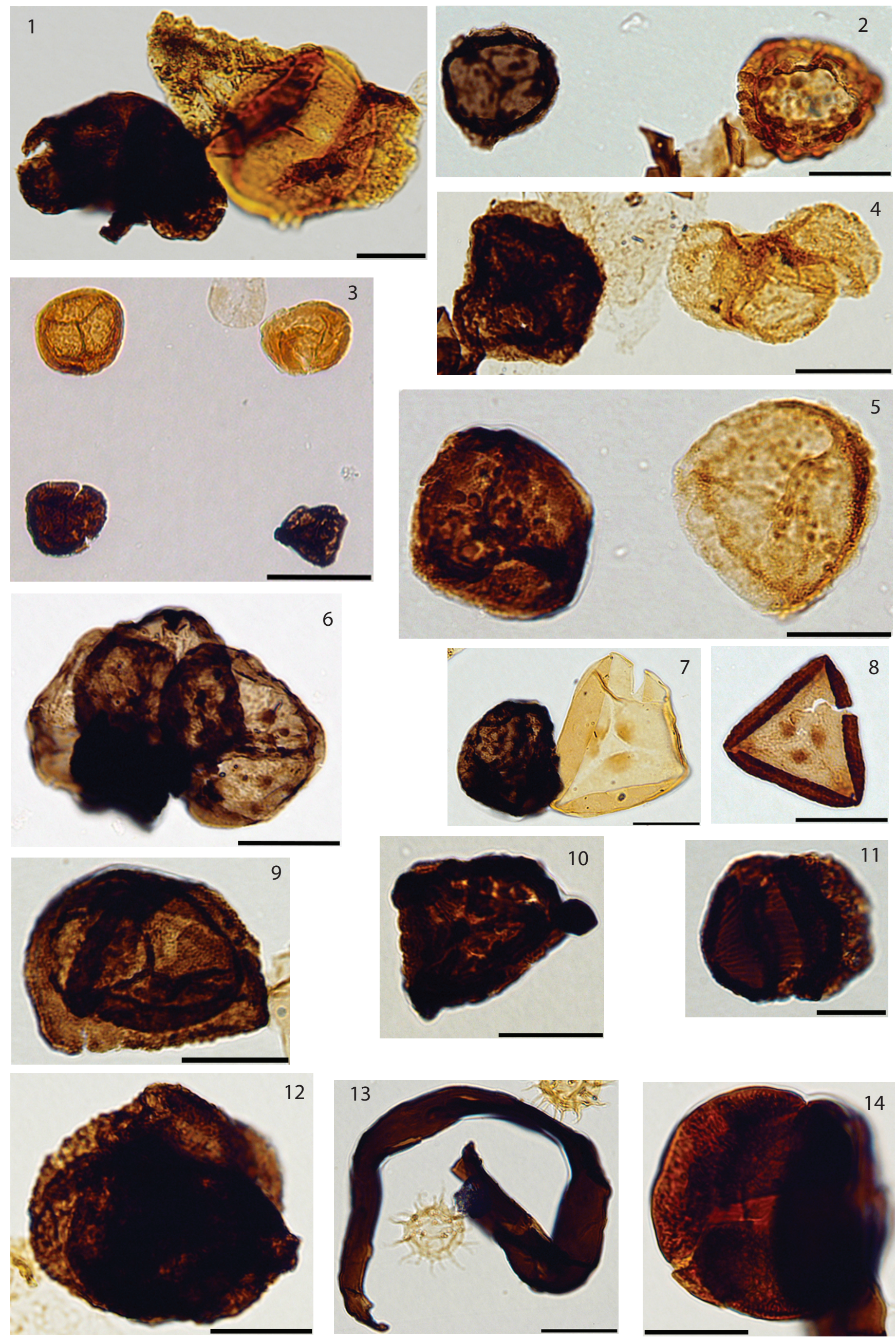
Table DR1

Raw counts

\begin{tabular}{rrrrrrrr} 
& $\begin{array}{r}\text { Undeformed } \\
\text { sporomorphs }\end{array}$ & $\begin{array}{r}\text { Indeterminate } \\
\text { sporomorphs* }\end{array}$ & tetrads & $\begin{array}{r}\text { deformed } \\
\text { tetrads }\end{array}$ & $\begin{array}{r}\text { Deformed } \\
\text { sporomorphs }\end{array}$ & $\begin{array}{r}\text { Darkened } \\
\text { sporomorphs }\end{array}$ & Sum \\
\hline 65.64 & 59 & 148 & 2 & 5 & 54 & 14 & 282 \\
67.86 & 20 & 100 & 3 & 3 & 48 & 18 & 192 \\
69.3 & 41 & 167 & 1 & 1 & 49 & 11 & 270 \\
73.39 & 40 & 170 & 2 & 2 & 35 & 21 & 270 \\
79.85 & 50 & 155 & 2 & 3 & 42 & 23 & 275 \\
81.87 & 40 & 148 & 1 & 7 & 51 & 26 & 273 \\
84.74 & 56 & 137 & 1 & 3 & 43 & 23 & 263 \\
85.95 & 26 & 193 & 1 & 2 & 46 & 36 & 304 \\
87.7 & 100 & 103 & 6 & 4 & 52 & 6 & 271 \\
90.96 & 67 & 152 & 0 & 0 & 50 & 4 & 273 \\
94.88 & 73 & 120 & 5 & 11 & 68 & 5 & 282 \\
100.04 & 69 & 114 & 9 & 7 & 58 & 4 & 261 \\
105.69 & 54 & 124 & 7 & 7 & 62 & 14 & 268 \\
108.79 & 63 & 113 & 1 & 8 & 66 & 21 & 272 \\
110.06 & 40 & 129 & 10 & 13 & 48 & 24 & 264 \\
112.76 & 71 & 116 & 5 & 8 & 62 & 20 & 282 \\
114.37 & 63 & 130 & 4 & 9 & 31 & 33 & 270 \\
115.29 & 17 & 168 & 6 & 21 & 48 & 28 & 288 \\
115.9 & 44 & 113 & 10 & 14 & 69 & 17 & 267 \\
116 & 22 & 125 & 3 & 16 & 71 & 10 & 247 \\
116.46 & 22 & 150 & 1 & 4 & 85 & 13 & 275 \\
116.47 & 29 & 129 & 0 & 2 & 109 & 3 & 272 \\
117.49 & 19 & 165 & 1 & 0 & 90 & 0 & 275 \\
119.09 & 35 & 133 & 0 & 0 & 130 & 0 & 298 \\
141.09 & 46 & 164 & 0 & 0 & 17 & 0 & 227
\end{tabular}

- * due to corrosion or insituable orientation 


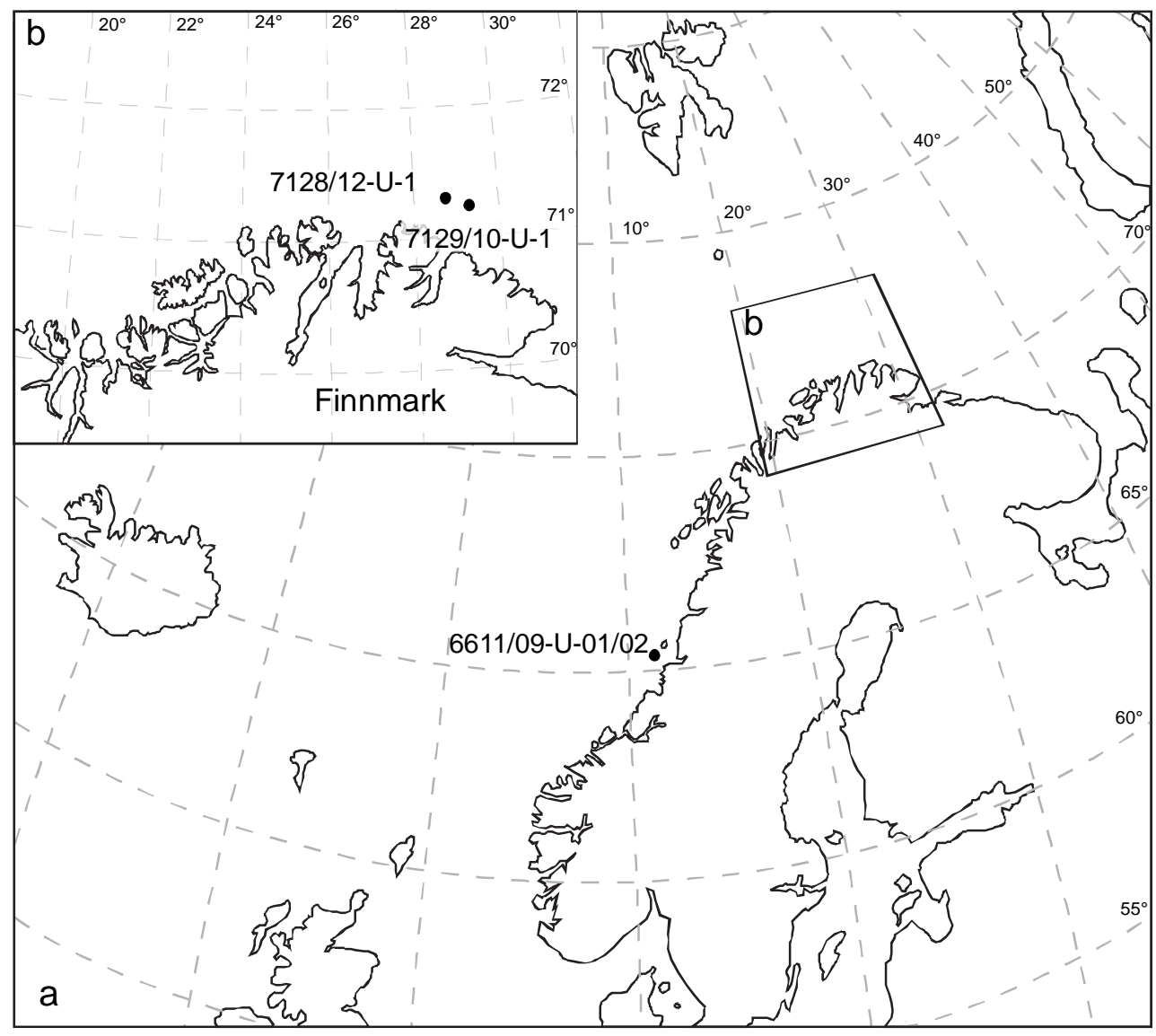

Figure DR1 : Location of core sites in Norway. 


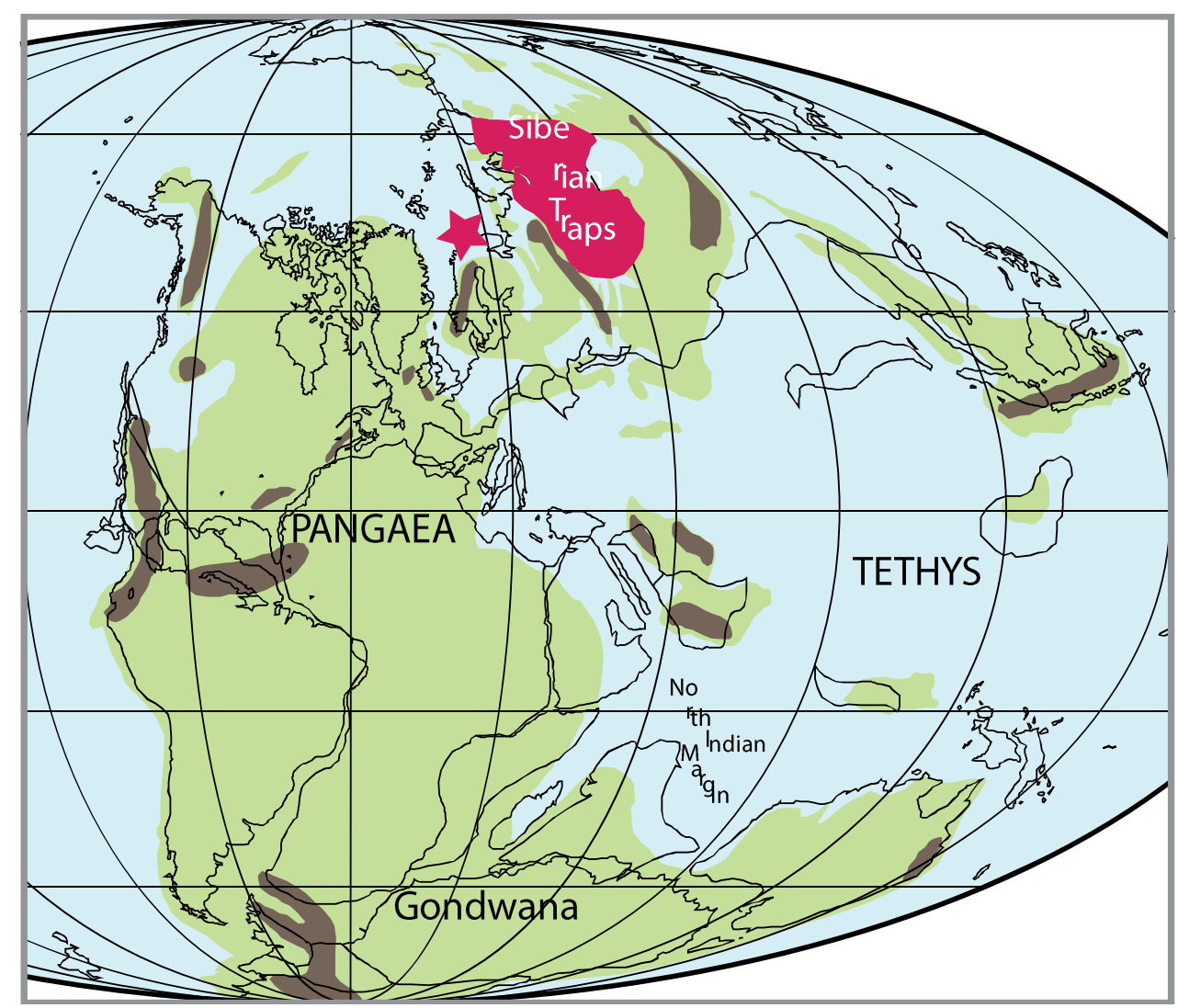

Figure DR2 : Permian-Triassic palaeogeography after Smith et al., 1994 and Golonka and Ford, 2000. Palaogeographic position of the Finnmark cores is indicated by a star. Palaegeographic position and extent of the Siberian Traps after Reichow et al., 2009

\section{Reference :}

Golonka, J. and Ford, D. 2000. Pangaean (Late Carboniferous-Middle Jurassic) paleoenvironment and lithofacies. Palaeogeography, Palaeoclimatoloy, Palaeoecology, 161, 1-34.

Reichow, M.K., Pringle, M.S., Al'Mukhamedov. A.I., Allen, M.B., Andreichev, V.L., Buslow, M.M., Davies, C.E., Fedoseev, G.S., Fitton, J.G., Inger, S., Medvedev, A.Ya., Mitchell, C., Puchkov, V.N., Safonova, I. Yu., Scott, R.A., Saunders, A.D. 2009. The timing and extent of the eruption of the Siberian traps large igneous province: implications for the end-Permian environmental crisis. Earth and Planetary Science Letters, 277, 9-20.

Smith, A.G., Smith, D.G., and Funnell, B.M., 1994, Atlas of Mesozoic and Cenozoic Coastlines: Cambridge, UK, Cambridge University Press, 109 p. 